\title{
ON THE DISCRETE SERIES OF GENERALIZED STIEFEL MANIFOLDS
}

\author{
JIAN-SHU LI
}

\begin{abstract}
A study of the discrete series of generalized Stiefel manifolds is made using the oscillator representation. New infinite families of such discrete series are constructed.
\end{abstract}

\section{INTRODUCTION}

Let $D$ be one of the three division algebras $\mathbf{R}, \mathbf{C}$ or $\mathbf{H}$ (the quaternion algebra) over $\mathbf{R}$, with their usual involutions. Let $V$ be a vector space over $D$, endowed with a nondegenerate hermitian form (, ). Let $G=U(V)$ be the group of isometries of $($,$) . Up to equivalence ($,$) is determined by$ its signature, say $(p, q)$. We write $G=U(p, q ; D)$, or simply $G=U(p, q)$ when $D$ is understood implicitly.

Fix a pair of integers $(r, s)$ with $0 \leq r \leq p, 0 \leq s \leq q$. We call the homogeneous space $U(p, q ; D) / U(p-r, q-s ; D)$ a generalized Stiefel manifold. This space carries a natural $G$-invariant measure, and hence there is a natural unitary representation of $G$ on $L^{2}(U(p, q ; D) / U(p-r, q-s ; D))$ via left translations. We say that an irreducible unitary representation of $G$ belongs to the discrete series of the (generalized) Stiefel manifold if it can be realized as a subrepresentation of $L^{2}(U(p, q ; D) / U(p-r, q-s ; D))$. The purpose of this paper is to introduce a new method for the study of this discrete series, and to construct new infinite families of discrete series for $U(p, q ; D) / U(p-r, q-s ; D)$. Let $n=r+s$. When $s=0, U(p, q ; D) / U(p-n, q ; D)$ is what is called an indefinite Stiefel manifold [12], and its discrete series were studied by Schlichtkrull [18] and Kobayashi [12]. The results of the present paper will show that as soon as $n>1$, the families of discrete series for $U(p, q ; D) / U(p-n, q ; D)$ constructed by Schlichtkrull and Kobayashi are not exhaustive. In fact there exist new infinite families of discrete series which are not in the lists of Schlichtkrull and Kobayashi.

Our understanding of the discrete series come from its relation with the oscillator representation. In $\S 2$ it will be shown that the study of discrete series of our generalized Stiefel manifolds is more or less equivalent to the study of the discrete spectrum of the oscillator representation associated to certain reductive dual pairs. This relationship was noticed before in special cases,

Received by the editors May 28, 1991 and, in revised form, October 3, 1991.

1991 Mathematics Subject Classification. Primary 22E45; Secondary 15A72.

Supported in part by NSF grant No. DMS-9003999. 
notably by Gelbart [7] for $O(m) / O(n)$ and by Rallis and Schiffmann [17] for $O(p, q) / O(p-1, q)$.

We would like to thank David Vogan for explaining the relationship between $N$-spectrum and asymptotic support, which is essential for $\S 4$. We thank T. Kobayashi and T. Uzawa for sending us a preprint which provided the stimulation for the present work.

\section{The oscillator RePRESENTATION AND Stiefel MANifoldS}

Let $G=U(p, q ; D)$ be as in $\S 1$ and fix an integer $n \leq p+q$. We endow the $D$-vector space $V^{\prime}=D^{n} \oplus\left(D^{n}\right)^{*}$ with a maximally split skew hermitian form $(,)^{\prime}$, and let $G^{\prime}$ be its group of isometries. Thus $G^{\prime} \simeq S p_{2 n}(\mathbf{R}), U(n, n)$ or $O^{*}(4 n)$. We identify $D^{n}$ and $\left(D^{n}\right)^{*}$ with the first and second summand of $V^{\prime}$, and assume that they are both totally isotropic subspaces of $V^{\prime}$. The form $(,)^{\prime}$ identifies $D^{n},\left(D^{n}\right)^{*}$ with duals of each other (hence the notation).

The pair of groups $G, G^{\prime}$ form a reductive dual pair [8] inside the symplectic group $S p_{2 m}(\mathbf{R})$, where

$$
m=n(p+q) \cdot \operatorname{dim}_{\mathbf{R}}(D) .
$$

Let $\omega$ denote an oscillator representation of $S p_{2 m}(\mathbf{R})$. There are essentially two such representations depending on the choice of a nontrivial character of $\mathbf{R}$; our choice here is specified by the formula (6) below. We also recall that $\omega$ is in fact a representation of the nontrivial two-fold cover of $S p_{2 m}(\mathbf{R})$. In the present setting, this two-fold cover is trivial when restricted to $G$. Hence the restriction of $\omega$ to $G$ can and will be considered an ordinary representation of $G$.

To simplify the notations we write $V^{n}=\operatorname{Hom}_{D}\left(D^{n}, V\right)$, the space of $D$ linear maps from $D^{n}$ to $V$. The representation $\omega$ can be realized on $L^{2}\left(V^{n}\right)$, where the action of $G$ is simply given by post-multiplication

$$
\omega(g) f(x)=f\left(g^{-1} x\right)
$$

for $g \in G, f \in L^{2}\left(V^{n}\right), x \in V^{n}$.

For each subgroup $E$ of $G^{\prime}$ we let $\widetilde{E}$ be the pre-image of $E$ in the two-fold cover of $S p_{2 m}(\mathbf{R})$. The action of $\widetilde{G}^{\prime}$ is less straightforward. Let $P$ be the stablizer of $D^{n}$ in $G^{\prime}$. This is a maximal parabolic subgroup of $G^{\prime}$. If $M$ denotes the subgroup of $P$ consisting of those linear maps which preserve both $D^{n}$ and $\left(D^{n}\right)^{*}$ then $M \simeq G L(n, D)$, and we have the Levi decomposition $P=M N$, where $N$ is the unipotent radical of $P$. If $m \in M$ and $\tilde{m}$ denotes a pre-image of $m$ in $\widetilde{G}^{\prime}$ then

$$
\omega(\tilde{m}) f(x)=\nu(\tilde{m}) f(x m) .
$$

Here $\nu(\tilde{m})$ is a complex number whose absolute value $|\nu(\tilde{m})|$ is determined by the fact that the action defined by (2) is unitary. Also $\nu(\tilde{m}) /|\nu(\tilde{m})|$ is at most a fourth root of unity.

The group $N$ can be lifted to a unique subgroup of $\widetilde{G}^{\prime}$; and that subgroup will be identified with $N$ itself. It is easy to see that $N$ is isomorphic to the space of hermitian forms on $\left(D^{n}\right)^{*}$, or equivalently, the space of $n \times n$ hermitian matrices with entries in $D$. We let $B\left(\left(D^{n}\right)^{*}\right)$ denote that space. For each $\beta \in B\left(\left(D^{n}\right)^{*}\right)$ we let $n_{\beta}$ be the corresponding element of $N$. Similarly 
we let $B\left(D^{n}\right)$ be the space of hermitian forms on $D^{n}$. Consider the natural involution

$$
\text { *: } \operatorname{End}_{D}\left(V^{\prime}\right) \rightarrow \operatorname{End}_{D}\left(V^{\prime}\right), \quad T \rightarrow T^{*},
$$

defined by the identity

$$
(T u, v)^{\prime}=\left(u, T^{*} v\right)^{\prime} \quad\left(u, v \in V^{\prime}\right) .
$$

This involution preserves $\operatorname{Hom}_{D}\left(D^{n},\left(D^{n}\right)^{*}\right)$, which is to be identified with the subspace of those endomorphisms of $V^{\prime}$ that vanish on $\left(D^{n}\right)^{*}$ and have their images contained in $\left(D^{n}\right)^{*}$. Similarly it preserves $\operatorname{Hom}_{D}\left(\left(D^{n}\right)^{*}, D^{n}\right)$. We have natural isomorphisms

$$
\begin{aligned}
B\left(\left(D^{n}\right)^{*}\right) & \simeq\left\{\beta \in \operatorname{Hom}_{D}\left(\left(D^{n}\right)^{*}, D^{n}\right) \mid \beta^{*}=\beta\right\}, \\
B\left(D^{n}\right) & \simeq\left\{\gamma \in \operatorname{Hom}_{D}\left(D^{n},\left(D^{n}\right)^{*}\right) \mid \gamma^{*}=\gamma\right\} .
\end{aligned}
$$

We identify $B\left(D^{n}\right)$ and $B\left(\left(D^{n}\right)^{*}\right)$ with linear duals of each other as follows. According to the above, given $\beta \in B\left(\left(D^{n}\right)^{*}\right)$ and $\gamma \in B\left(D^{n}\right)$, the composite $\beta \gamma$ is an endomorphism of $D^{n}$. Let tr denote the map which takes a matrix to the sum of its diagonal entries, followed by the trace map of $D$ over $\mathbf{R}$. The bilinear form

$$
\langle\beta, \gamma\rangle=\operatorname{tr}(\beta \gamma)
$$

exhibits the duality involved.

We now consider the orbit parameter map

$$
\tau: V^{n}=\operatorname{Hom}_{D}\left(D^{n}, V\right) \rightarrow B\left(D^{n}\right)
$$

where for $x \in \operatorname{Hom}_{D}\left(D^{n}, V\right), \tau(x)$ is the hermitian form defined by

$$
\tau(x)(u, v)=(x(u), x(v)) \quad\left(u, v \in D^{n}\right) .
$$

The action of $N$ on $L^{2}(X)$ is now given by

$$
\omega\left(n_{\beta}\right) f(x)=\psi(\operatorname{tr}(\tau(x) \beta)) f(x) \quad\left(\beta \in B\left(\left(D^{n}\right)^{*}\right)\right) .
$$

Here $\psi$ is the character defined by $\psi(x)=e^{2 \pi i x}$.

The group $G$ acts on $V^{n}$ by post-multiplications. Its generic orbits are classified by the orbit parameter map (4). More precisely we have the following version of Witt's theorem:

Lemma 2.1. Let $x, x^{\prime} \in V^{n}$. If $\tau(x)=\tau\left(x^{\prime}\right)$ and this hermitian form is nondegenerate then there is a $g \in G$ such that $g x=x^{\prime}$.

On the other hand, $M=G L(n, D)$ acts on $V^{n}$ via pre-multiplication; and there is also a natural action of $M$ on $B\left(D^{n}\right)$ so that the orbit parameter map $\tau$ is $M$-equivariant. An $M$-orbit on $B\left(D^{n}\right)$ is nothing but an equivalence class of hermitian forms on $D^{n}$. Let $r, s$ be integers with $0 \leq r \leq p, 0 \leq s \leq q$ and $r+s=n$. Set

$$
\Omega_{r, s}=\left\{x \in V^{n} \mid \tau(x) \text { is of signature }(r, s)\right\} .
$$

The above discussion shows that under the action of $G \times M$, the open orbits in $V^{n}$ are precisely the sets $\Omega_{r, s}$ defined above. It follows that we have the direct sum decomposition

$$
L^{2}\left(V^{n}\right)=\bigoplus L^{2}\left(\Omega_{r, s}\right), \quad(0 \leq r \leq p, 0 \leq s \leq q, r+s=n) .
$$


From (1), (2) and (6) we see that each summand in the above decomposition is stable under $G$ and under $\widetilde{P}$.

Fix a pair of integers $r, s$ as above and choose $X_{0} \in \Omega_{r, s}$. We get a fibration

$$
G \times M \rightarrow \Omega_{r, s}, \quad(g, m) \rightarrow g X_{0} m .
$$

Let $V_{0} \subseteq V$ be the image of $X_{0}$ and let $H$ be the stabilizer of $V_{0}$ in $G$. One has $H=H_{1} \times H_{2}$ with $H_{1}=U\left(V_{0}\right) \simeq U(r, s ; D), H_{2}=U\left(V_{0}^{\perp}\right)=$ $U(p-r, q-s ; D)$. Note that $X_{0}$ defines a linear isomorphism from $D^{n}$ to $V_{0}$; hence $X_{0}^{-1}: V_{0} \rightarrow D^{n}$ is defined.

Lemma 2.2. Let $g \in G, m \in M$. If $g X_{0} m=X_{0}$ then $g=h_{1} h_{2}$ with $h_{1} \in H_{1}$, $h_{2} \in H_{2}$ and $m=X_{0}^{-1} h_{1}^{-1} X_{0}$.

Proof. We have $\tau\left(X_{0}\right)=\tau\left(g X_{0} m\right)=\tau\left(X_{0} m\right)$, which means $m$ is in the isometry group of $\tau\left(X_{0}\right)$. Hence $m=X_{0}^{-1} h X_{0}$ with $h \in H_{1}$. Now $X_{0}=g h X_{0}$, hence $g h \in H_{2}$, and the lemma follows by taking $h_{1}=h^{-1}, h_{2}=g h$.

If $h \in H_{2}$ then $h X_{0}=X_{0}$, and hence (9) induces a fibration

$$
\phi: G / H_{2} \times M \rightarrow \Omega_{r, s}
$$

where each fiber is isomorphic to $H_{1}$. In other words we have

$$
\Omega_{r, s} \simeq G / H_{2} \times_{H_{1}} M \text {. }
$$

Set $K=X_{0}^{-1} H_{1} X_{0}$. This is a closed subgroup of $M$. Let $\left\{M_{i}^{\prime}\right\}$ be a countable family of left $K$-invariant open subsets of $M$ whose union is all of $M$, such that for each $i$ there is a subset $R_{i}^{\prime}$ of $M$ with $M_{i}^{\prime}=K R_{i}^{\prime}$, and that the map $K \times R_{i}^{\prime} \rightarrow M_{i}^{\prime}$ is an isomorphism (of topological spaces). Now set

$$
M_{1}=M_{1}^{\prime}, \quad M_{i+1}=M_{i+1}^{\prime}-\bigcup_{j \leq i} M_{j}^{\prime} .
$$

Then $M$ is the disjoint union of the $M_{i}$ 's and for each $i$ we have $M_{i}=K R_{i}$ for some $R_{i} \subseteq R_{i}^{\prime}$. By Lemma 2.2 we have

$$
G / H_{2} \times_{H_{1}} M_{i} \simeq G / H_{2} \times R_{i} .
$$

Hence (11) implies

$$
L^{2}\left(\Omega_{r, s}\right)=\bigoplus_{i} L^{2}\left(G / H_{2} \times R_{i}\right)
$$

where on the right-hand side $\mathrm{G} / \mathrm{H}_{2}$ is endowed with the unique (up to constants) $G$-invariant measure, while the measure on $R_{i}$ comes from an $M$ invariant measure on $K \backslash M$.

There is one case where the above decomposition is particularly simple: suppose $r=0$ or $s=0$. Then $K$ is a maximal compact subgroup of $M$. Let $M=K A N$ be an Iwasawa decomposition. Then (11) gives $\Omega_{r, s} \simeq G / H_{2} \times A N$.

Theorem 2.3. The unitary representation of $G$ on $L^{2}\left(\Omega_{r, s}\right)$ is a multiple of $L^{2}\left(G / H_{2}\right)$.

Theorem 2.4. The restriction of the oscillator representation $\omega$ to $G=U(p, q ; D)$ is the direct sum of $L^{2}(U(p, q ; D) / U(p-r, q-s ; D)), r \leq p, s \leq q, r+s=n$, with multiplicities.

These are obvious consequences of (8) and (12). 


\section{3. $L^{p}$-PROPERTIES}

Let $\rho$ be a unitary representation of $G$ realized on the Hilbert space $H_{\rho}$.

Definition 3.1. Let $p>0$ be a real number. We say that $\rho$ is strongly $L^{p}$ if there is a dense subset $H \subseteq H_{\rho}$ such that for any $\xi, \eta \in H$, the associated matrix coefficient $x \rightarrow(\rho(x) \xi, \eta)$ belongs to $L^{p}$. We say that $\rho$ is strongly $L^{p+\varepsilon}$ if it is strongly $L^{p^{\prime}}$ for any $p^{\prime}>p$.

Definition 3.2. The spectrum of $\rho$ is the set of irreducible unitary representations of $G$ weakly contained in $\rho$. $\S 2)$.

Let $\omega_{n}=\left.\omega\right|_{G}$ be the restriction to $G$ of the oscillator representation (cf.

Lemma 3.3. (a) The representation $\omega_{n}$ is strongly $L^{p_{n}+\varepsilon}$, where

$$
p_{n}=\frac{1}{n}\left(p+q-\frac{2}{\operatorname{dim}_{\mathbf{R}} D}\right) .
$$

(b) The restriction of $\omega$ to $G^{\prime}$ is strongly $L^{r}$ for any $r$ satisfying

$$
r>\frac{4}{p+q}\left(n-1+\frac{1}{\operatorname{dim}_{\mathbf{R}} D}\right) .
$$

Proof. See [13, Theorem 3.2].

Theorem 3.4. Let $2 k$ be the smallest even integer satisfying

$$
2 k \geq \frac{1}{n}\left(p+q-\frac{2}{\operatorname{dim}_{\mathbf{R}} D}\right) .
$$

Let

$$
\omega_{n}=\int_{X}^{\oplus} \pi_{x} d \mu(x)
$$

be the direct integral decomposition of $\omega_{n}$ into irreducible unitary representations of $G$. Then for almost all $x$, the representation $\pi_{x}$ is strongly $L^{2 k+\varepsilon}$.

Proof. Let $K$ and $P_{1}$ be a maximal compact subgroup and a minimal parabolic subgroup of $G$, such that the Iwasawa decomposition $G=K P_{1}$ holds. Let $\delta$ be the left modular function of $P_{1}$. Extend $\delta$ to a function on $G$ by the formula $\delta(k p)=\delta(p)$ for $k \in K, p \in P_{1}$. Let $\Xi$ be the function defined by Harish-Chandra

$$
\Xi(x)=\int_{K} \delta^{-1 / 2}(x k) d k \quad(x \in G) .
$$

Let $\mu$ be an irreducible representation of $K$ and $\xi, \eta$ vectors in the space of $\omega_{n}$ which transforms under $K$ according to a multiple of $\mu$. By the corollary on [5, p. 108], we have for any $x \in G$,

$$
\left|\left(\omega_{n}(x) \xi, \eta\right)\right| \leq(\operatorname{dim} \mu)^{2}\|\xi\|\|\eta\| \Xi^{1 / k}(x) .
$$

If $\omega_{n}$ is decomposed into a direct integral as stated in the theorem then the same estimate (16) is valid for almost all $\pi_{x}$. But, as the Harish-Chandra function $\Xi \in L^{2+\varepsilon}(G)$, this implies that for almost all $x, \pi_{x}$ is strongly $L^{2 k+\varepsilon}$. Q.E.D. 
Remark. The appearance of the even integer $2 k$ is an artifact resulting from the technical difficulties involved in the proof of [5]. Let $p_{n}$ be as defined by (13). It is natural to wonder whether in the above theorem the representations $\pi_{x}$ (for almost all $x$ ) are in fact strongly $L^{p_{n}+\varepsilon}$, as long as $p_{n} \geq 2$. Such an estimate would then be sharp, as can be seen from the next example.

Example. Consider the case $D=\mathbf{R}, q=1$. Then $G=O(p, 1), G^{\prime}=$ $S p_{2 n}(\mathbf{R})$. Let $G_{0}$ be the connected component of the identity in $G$. The spherical complementary series of $G_{0}$ may be parametrized by the interval $0 \leq \nu \leq \rho$ with $\rho=\frac{p-1}{2}$. Let $\pi_{\nu}$ be the representation corresponding to $\nu$. Then $\pi_{0}$ is a tempered representation while $\pi_{\rho}$ is the trivial one. For $0<\nu<\rho, \pi_{\nu}$ is strongly $L^{(p-1) /(\rho-\nu)+\varepsilon}$ but not strongly $L^{(p-1) /(\rho-\nu)}$. Suppose $n<(p-1) / 2$ then it can be shown that $\pi_{\rho-n}$ is a subrepresentation of (the restriction of) $\omega_{n}$. Note that $p_{n}=(p-1) /(\rho-\nu)$ here!

Lemma 3.5. Let $\tau$ be a strongly $L^{2}$ representation on the Hilbert space $H$. Let $\pi$ be an irreducible subrepresentation of $\tau$. Then $\pi$ belongs to the discrete series of $G$.

Proof. Let $H_{\pi} \subseteq H$ be the closed subspace affording the representation $\pi$. There is a dense subspace $H_{0} \subseteq H$ such that all matrix coefficients associated to vectors in $H_{0}$ are in $L^{2}(G)$. We fix a vector $v_{0} \in H_{0}$ which is not orthogonal to $H_{\pi}$. The map

$$
\phi: H \rightarrow L^{2}(G)
$$

which takes $v \in H$ to the matrix coefficient $\left(\tau(x) v, v_{0}\right)$ is a densely defined $G$-intertwining operator with a closable graph. By the general Schur's lemma [2], we get a unitary embedding of $(\operatorname{ker} \phi)^{\perp}$ into $L^{2}(G)$. Since $H_{\pi}$ is not orthogonal to $v_{0}$ and $\pi$ is irreducible, we see that $H_{\pi} \subseteq(\operatorname{ker} \phi)^{\perp}$. Thus $\pi$ is an irreducible subrepresentation of $L^{2}(G)$. Q.E.D.

Theorem 3.6. There is a one-to-one correspondence $\pi \rightarrow \sigma$ between irreducible subrepresentations of $\omega_{n}$ and $\left.\omega\right|_{\widetilde{G}^{\prime}}$. The representations $\pi$ and $\sigma$ are Howe duals of each other in the sense of [8]. Furthermore, if $p+q \leq 2 n$ then $\pi$ belongs to the discrete series of $G$, i.e., it is square integrable. If $p+q \geq 2 n$ then $\sigma$ is square integrable.

Proof. The one-to-one correspondence is established in [9]. Let $H$ be the largest closed subspace of $L^{2}\left(V^{n}\right)$ on which $G$ acts by a multiple of $\pi$. Then $H$ is stable under $G$ and $\widetilde{G}^{\prime}$. By $[9, \S 7]$, the joint action of $G \times \widetilde{G}^{\prime}$ on $H$ is of the form $\pi \otimes \sigma$, where $\sigma$ is an irreducible unitary representation of $\widetilde{G}^{\prime}$. In particular, $\sigma$ is an irreducible subrepresentation of $\left.\omega\right|_{\widetilde{G}^{\prime}}$. Assume $p+q \leq 2 n$. We have $p_{n}<2$ in this case. According to Lemma 3.3, $\omega_{n}$ is strongly $L^{2}$. So $\pi$ is square integrable by Lemma 3.5. By [14, $\S 2]$, we see that $\pi$ and $\sigma$ are the Howe duals of each other in the sense defined in [8].

The proof in the case $p+q \geq 2 n$ is similar by reversing the role of $G$ and $G^{\prime}$. Note that in this case $\left.\omega\right|_{\widetilde{G}^{\prime}}$ is strongly $L^{2}$ unless $p+q=2 n$ and $D=\mathbf{R}$. However in this special case our result is valid by [16] or [14]. Q.E.D.

Combined with results obtained in $\S 2$, we immediately deduce the following results about Stiefel manifolds. 
Theorem 3.7. Let $2 k$ be the smallest even integer satisfying (15). Let

$$
L^{2}(U(p, q ; D) / U(p-r, q-s ; D))=\int_{X}^{\oplus} \pi_{x} d \mu(x)
$$

be the direct integral decomposition into irreducible unitary representations of $G$. Then for almost all $x$, the representation $\pi_{x}$ is strongly $L^{2 k+\varepsilon}$.

Corollary 3.8. Suppose $p+q \leq 2 n$ then the spectrum of

$$
L^{2}(U(p, q ; D) / U(p-r, q-s ; D))
$$

consists of tempered representations.

Theorem 3.9. Let $\pi$ be an irreducible unitary representation of $G$ belonging to the discrete series of $U(p, q ; D) / U(p-r, q-s ; D)$. There is a unique irreducible unitary representation $\sigma$ of $\widetilde{G}^{\prime}$ which corresponds to $\pi$ via Howe duality. Suppose $p+q \leq 2 n$. Then $\pi$ belongs to the discrete series of $G$ itself; while if $p+q \geq 2 n$ then $\sigma$ is square integrable.

Corollary 3.10. Let $s=0$. Suppose $p+q \leq 2 n$ and $n \leq p$. Then the discrete series of the symmetric space $U(p, q ; D) / U(n) \times U(p-n, q ; D)$ must be contained in the discrete series of $U(p, q ; D)$.

Corollary 3.11. Take $D=\mathbf{R}$ so that $U(p, q ; D)=O(p, q)$. Suppose $p, q$ are both odd and $p+q \leq 2 n$. Then $O(p, q) / O(p-r, q-s)$ contains no discrete series. If also $n \leq p$ then the symmetric space $O(p, q) / O(n) \times O(p-n, q)$ contains no discrete series.

Of course Corollary 3.10 and the last sentence of Corollary 3.11 would also follow from the known description of discrete series for symmetric spaces [15].

\section{THE $N$-SPECTRUM AND DISCRETE SERIES}

Definition 4.1. If $\pi$ and $\sigma$ are as in Theorem 3.7 we write $\pi=\theta(\sigma)$ and $\sigma=\theta(\pi)$. We let $\theta(\sigma)=0$ if $\sigma$ does not occur in Howe's correspondence.

In this section we assume $p+q \geq 2 n$. Let $\sigma$ be an irreducible square integrable representation of $\widetilde{G}^{\prime}$. By $\S 3$ we know that $\theta(\sigma)$ is in the discrete series of $U(p, q) / U(p-r, q-s)$ for some $r, s$ with $r+s=n$. Our task here is to determine exactly which $r, s$. The answer to this lies in the notion of $N$-spectrum introduced in [10].

Consider the parabolic $P=M N$ of $G^{\prime}$ introduced in $\S 2$. Let $\widehat{N}$ be the Pontrjagin dual of $N$. We have $N \simeq B\left(\left(D^{n}\right)^{*}\right)$ (cf. $\S 2$ ) and we can identify $B\left(D^{n}\right)$ with $\widehat{N}$, by associating to $\gamma \in B\left(D^{n}\right)$ the character $\psi_{\gamma}$ defined by

$$
\psi_{\gamma}(x)=\psi(\operatorname{tr}(x \gamma)) .
$$

Here $\psi$ and other things are as in (6). Let $\sigma$ be a unitary representation of $P$. By the direct integral theory [6], the restriction $\left.\sigma\right|_{N}$ is determined up to unitary equivalence by a projection valued measure $\pi_{\sigma}$ on $\widehat{N}$. This projection valued measure, and the unitary equivalence class it defines, is called the $N$-spectrum of $\sigma$ (see [10, §2]). Let $\mathscr{O}$ be an $\operatorname{Ad}^{*} P$-orbit in $\widehat{N}$. As explained in [10], the restriction of $\pi_{\sigma}$ to $\mathscr{O}$ is of uniform multiplicity, and the $N$-spectrum of $\sigma$ is determined by these multiplicities for all the orbits. Following [10], we say that 
an orbit $\mathscr{O}$ occurs in $\sigma$ if its multiplicity is nonzero. We say $\sigma$ is supported on the orbits which occur in $\sigma$. Via the identification of $\widehat{N}$ with $B\left(D^{n}\right)$, an $\operatorname{Ad}^{*} P$-orbit is the same thing as an equivalence class of hermitian forms on $D^{n}$, which is determined by the signature, say $(r, s)$, of any form in the class. We denote by $\mathscr{O}_{r, s}$ the orbit corresponding to forms of signature $(r, s)$. We have $r+s \leq n$ and $\mathscr{O}_{r, s}$ is open in $\widehat{N}$ if and only if $r+s=n$. Finally, if $\sigma$ is a representation of $\tilde{G}^{\prime}$ we can restrict it first to $\widetilde{P}$ and then to $N$. Hence the notion of $N$-spectrum applies to $\sigma$. We let $N S(\sigma)$ be the union of the $\operatorname{Ad}^{*} P$-orbits which occur in $\sigma$.

Note that the decomposition (8) is preserved by $\widetilde{P}$. By (6) we have

Lemma 4.2. In (8) the $N$-spectrum of the representation of $\widetilde{P}$ on $L^{2}\left(\Omega_{r, s}\right)$ is supported on the open orbit $\mathscr{O}_{r, s}$.

Let $\sigma$ be an irreducible square integrable representation for $\widetilde{G}^{\prime}$. By the above lemma and (8), (12), we see that $\theta(\sigma)$ occurs in $L^{2}(U(p, q) / U(p-r, q-s))$ if and only if $\mathscr{O}_{r, s} \subseteq N S(\sigma)$. Write $\widehat{\widetilde{G}}_{d}^{\prime}$ for the set of (equivalence classes of) irreducible square integrable representations of $\widetilde{G}^{\prime}$. We have

Theorem 4.3. The discrete series of $U(p, q) / U(p-r, q-s)$ is precisely the set

$$
\left\{\theta(\sigma) \mid \sigma \in \widehat{\widetilde{G}}_{d}^{\prime}, \mathscr{\sigma}_{r, s} \subseteq N S(\sigma)\right\} .
$$

To complete the description of the discrete series of $U(p, q) / U(p-r, q-s)$, it remains to

(a) calculate the $N$-spectrum of $\sigma \in \widehat{\widetilde{G}}_{d}^{\prime}$;

(b) determine the parameter of $\theta(\sigma)$, here parameter means say the Langlands-Vogan parameter.

To a large degree, the answer to the first question is essentially known, at least conjecturely. The rest of this section will be devoted to a discussion of problem (a).

Let $\sigma$ be a representation of $\widetilde{G}^{\prime}$ on a Hilbert space $H$. We recall from [11] the notion of the wave front set of $\sigma$. Let $J_{1}(H)$ be the trace class operators on $H$. Given $T \in J_{1}(H)$, we let $\operatorname{tr}_{\sigma}(T)$ be the distribution defined by

$$
\operatorname{tr}_{\sigma}(T)(f)=\int_{\widetilde{G}^{\prime}} f(g) \operatorname{tr}(\sigma(g) T) d g
$$

We define $W F(\sigma)$ to be the closure of the union of the wave front sets at 0 for all $T \in J_{1}(H)$. (In the notation of [11], $W F(\sigma)$ is called $W F_{\sigma}^{0}$.) Let $\mathbf{g}$ be the Lie algebra of $G^{\prime}$. It is known that $W F(\sigma)$ is a union of nilpotent co-adjoint orbits in $\mathbf{g}^{*}$. Now suppose $\sigma$ has a distribution character $\Theta_{\sigma}$ and let $A S\left(\Theta_{\sigma}\right)$ be its asymptotic support, as defined in [3]. By [11 and 4, p. 159], we have the relation

$$
A S\left(\boldsymbol{\Theta}_{\sigma}\right) \subseteq W F(\sigma)
$$

(Presumably this should be an equality; however no written proof is known to the author.)

Let $\mathbf{n}$ be the Lie algebra of $N$. Then $\mathbf{n} \simeq B\left(\left(D^{n}\right)^{*}\right)$ and via the formula (17) we have $\widehat{N} \simeq \mathbf{n}^{*} \simeq B\left(D^{n}\right)$. We will use the following relation without proof, 
since it is implicit in [11]: Let $\overline{N S(\sigma)}$ be the closure of $N S(\sigma)$ in $\mathbf{n}^{*}$. Then

$$
W F\left(\left.\sigma\right|_{N}\right)=\overline{N S(\sigma)} \text {. }
$$

Let

$$
q: \mathbf{g}^{*} \rightarrow \mathbf{n}^{*}
$$

be the restriction map. By [11, Proposition 1.5] we have

$$
q(W F(\sigma)) \subseteq W F\left(\left.\sigma\right|_{N}\right) .
$$

This together with (19)-(20) gives

$$
q\left(A S\left(\Theta_{\sigma}\right)\right) \subseteq \overline{N S(\sigma)} .
$$

It is this formula that we will use to get some information about $N S(\sigma)$, at least when $\sigma$ is square-integrable.

Fix a Cartan decomposition $\mathbf{g}=\mathbf{k}+\mathbf{p}$. Let $\mathbf{t} \subseteq \mathbf{k}$ be a Cartan subalgebra. Choose compatible orderings for the root systems $\Delta\left(\mathbf{k}_{\mathbf{c}}, \mathbf{t}_{\mathbf{c}}\right)$ and $\Delta\left(\mathbf{g}_{\mathbf{c}}, \mathbf{t}_{\mathbf{c}}\right)$ (here the subscript $\mathbf{c}$ indicates complexifications). Let $\mathbf{t}^{+}$be the set of regular elements in a positive Weyl chamber for $\mathbf{k}$ and assume $\mathbf{t}^{+}=\bigcup_{j=1}^{s} \mathbf{t}^{j}$, where $\mathbf{t}^{j}$ are positive chambers for $\mathbf{g}$.

Let now $\sigma$ be a discrete series representation of $\tilde{G}^{\prime}$ with Harish-Chandra parameter $\lambda \in \mathbf{t}^{*}$. We write $\sigma=\sigma_{\lambda}$ and let $\Theta_{\lambda}$ be its distribution character. We choose a nondegenerate symmetric bilinear invariant form $B($,$) on \mathbf{g}$, by means of which we identify $\mathbf{g}$ with $\mathbf{g}^{*}$. Then $\lambda$ can be identified with a $Z_{\lambda} \in \mathbf{t}^{j}$. By a result of Barbasch and Vogan [3, Proposition 3.7], we have

$$
\begin{array}{r}
A S\left(\Theta_{\lambda}\right)=\left\{X \in \mathbf{g} \mid \text { there exist } x_{i} \in G^{\prime} \text { and } t_{i} \in \mathbf{R} \text { with } t_{i} \rightarrow 0,\right. \\
\text { such that } \left.X=\lim t_{i} \operatorname{Ad} x_{i} Z_{\lambda}\right\} .
\end{array}
$$

Further, $A S\left(\boldsymbol{\Theta}_{\lambda}\right)$ depends only on the chamber $\mathbf{t}^{j}$ which contains $Z_{\lambda}$.

Let $\mathbf{N}_{-}$be the unipotent radical of the parabolic opposite to $P$ and let $\mathbf{n}_{-}$be its Lie algebra. Then under the identification of $\mathbf{g}$ with $\mathbf{g}^{*}$ we have $\mathbf{n}_{-}=\mathbf{n}^{*} \simeq B\left(D^{n}\right)$ and the projection $q$ is identified with a map $q: \mathbf{g} \rightarrow \mathbf{n}_{-}$. We now realize elements of $\mathbf{g}$ as $2 n \times 2 n$ matrices. Write $M_{n}(D)$ for the set of $n \times n$ matrices with entries in $D$. We have

$$
\begin{gathered}
\mathbf{g}=\left\{\left(\begin{array}{cc}
A & B \\
C & -{ }^{t} \bar{A}
\end{array}\right) \mid A, B, C \in M_{n}(D),{ }^{t} \bar{B}=B,{ }^{t} \bar{C}=C\right\}, \\
\mathbf{n}=\left\{\left(\begin{array}{ll}
0 & 0 \\
C & 0
\end{array}\right) \mid{ }^{t} \bar{C}=C\right\} .
\end{gathered}
$$

The projection $q: \mathbf{g} \rightarrow B\left(D^{n}\right)=\mathbf{n}_{-}$is then given by the formula

$$
q\left(\begin{array}{cc}
A & B \\
C & -{ }^{t} \bar{A}
\end{array}\right)=B \text {. }
$$

We take

$$
\mathbf{k}=\left\{\left(\begin{array}{cc}
A & B \\
-B & A
\end{array}\right) \mid{ }^{t} \bar{A}=-A,{ }^{t} \bar{B}=B\right\} .
$$

Let $i, j, k$ be the standard basis for the quarternion algebra so that $\mathbf{H}=$ $\mathbf{R} \oplus \mathbf{R} i \oplus \mathbf{R} j \oplus \mathbf{R} k$. We consider $\mathbf{R} \subseteq \mathbf{C}=\mathbf{R} \oplus \mathbf{R} i \subseteq \mathbf{H}$. For the Cartan subalgebra $\mathbf{t}$ we take the set of matrices in $\mathbf{k}$ where $A, B$ are both diagonal, and that the entries of $A$ and $B$ are in $\mathbf{R} i$ and $\mathbf{R}$ respectively. 
We write

$$
Z_{\lambda}=\left(\begin{array}{cc}
R & S \\
-S & R
\end{array}\right)
$$

where $R, S$ are diagonal matrices as just described. In order to calculate the set $A S\left(\boldsymbol{\theta}_{\lambda}\right)$ given by (23), it is clearly sufficient to take the $x_{i}$ there in a fixed open dense subset of $G^{\prime}$. Now $N_{-} P$ is open dense in $G^{\prime}$, and if $x=n_{-} p$ with $n_{-} \in \mathbf{N}_{-}$and

$$
p=\left(\begin{array}{cc}
A & 0 \\
0 & { }^{t} \bar{A}^{-1}
\end{array}\right)\left(\begin{array}{cc}
1 & B \\
0 & 1
\end{array}\right) \in P
$$

we have

$$
\operatorname{Ad}(x)\left(Z_{\lambda}\right)=\left(\begin{array}{c}
* \quad A(S+B R-R B+B S B)^{t} \bar{A} \\
*
\end{array}\right)
$$

and hence by (24) we have

$$
q\left(\operatorname{Ad}(x)\left(Z_{\lambda}\right)\right)=A(S+B R-R B+B S B)^{t} \bar{A} .
$$

Let $\mathscr{O}_{\beta}$ denote the orbit, or equivalently the equivalence class, of $\beta \in B\left(D^{n}\right)$. Suppose $\mathscr{O}_{\beta}$ is open in $B\left(D^{n}\right)$. We see that in order for $\mathscr{O}_{\beta} \subseteq q\left(A S\left(\Theta_{\lambda}\right)\right)$, it is necessary that $\beta$ be equivalent to a hermitian form of the form given by the right-hand side of (26). Conversely, it is trivially seen that this is also sufficient. We obtain

Proposition 4.4. The open orbit $\mathscr{O}_{\beta}$ is contained in $q\left(A S\left(\boldsymbol{\Theta}_{\lambda}\right)\right)$ if and only if there is a hermitian matrix $B$ such that

$$
\beta \simeq S+B R-R B+B S B .
$$

Here $\simeq$ denotes equivalence of hermitian forms.

Note that an open orbit in $\overline{N S\left(\sigma_{\lambda}\right)}$ is necessarily contained in $N S\left(\sigma_{\lambda}\right)$. From (22) we have

Corollary 4.5. Let $Z_{\lambda}$ be given by (25). Suppose there is a hermitian matrix $B$ such that $S+B R-R B+B S B$ has signature $(r, s)$, where $r+s=n$. Then the orbit $\mathscr{O}_{r, s} \subseteq N S\left(\sigma_{\lambda}\right)$.

\section{CONSTRUCTION OF SOME DISCRETE SERIES}

We now write $S=\operatorname{diag}\left(s_{1}, \ldots, s_{n}\right), R=i \cdot \operatorname{diag}\left(r_{1}, \ldots, r_{n}\right)$ and assume

$$
s_{1}, \ldots, s_{k}>0>s_{k+1}, \ldots, s_{n} \text {. }
$$

By taking $B=0$ in (27) we see $\mathscr{O}_{k, l} \subseteq N S\left(\sigma_{\lambda}\right)$, where $l=n-k$.

Proposition 5.1. Let $m_{+}(S)$ (resp. $m_{-}(S)$ ) be the largest integer $t$ for which there are sequences of integers $0<i_{1}, \ldots, i_{t} \leq k$ and $k<j_{1}, \ldots, j_{t} \leq n$, such that $s_{i_{\mu}}+s_{j_{\mu}}>\left|r_{i_{\mu}}-r_{j_{\mu}}\right|$ (resp. $\left.s_{i_{\mu}}+s_{j_{\mu}}<-\left|r_{i_{\mu}}-r_{j_{\mu}}\right|\right)$ for $\mu=1, \ldots, t$. Then

$$
N S\left(\sigma_{\lambda}\right) \supseteq \mathscr{O}_{k+\mu, l-\mu} \text { for }-m_{-}(S) \leq \mu \leq m_{+}(S) .
$$

Proof. Consider first the case $n=2$. We assume $s_{1}>0>s_{2}$ (this being the only nontrivial case). We already know $\mathscr{O}_{1,1} \subseteq N S\left(\sigma_{\lambda}\right)$. Taking $B=\left(\begin{array}{ll}0 & 1 \\ 1 & 0\end{array}\right)$ in (27) we obtain

$$
S+B S B+B R-R B=\left(\begin{array}{cc}
s_{1}+s_{2} & -i\left(r_{1}-r_{2}\right) \\
i\left(r_{1}-r_{2}\right) & s_{1}+s_{2}
\end{array}\right)
$$


which is positive definite or negative definite according as $s_{1}+s_{2}>\left|r_{1}-r_{2}\right|$ or $s_{1}+s_{2}<-\left|r_{1}-r_{2}\right|$. Hence by Corollary 4.5 we have $\mathscr{O}_{2,0} \subseteq N S\left(\sigma_{\lambda}\right)$ or $\mathscr{O}_{0,2} \subseteq N S\left(\sigma_{\lambda}\right)$ according as $s_{1}+s_{2}>\left|r_{1}-r_{2}\right|$ or $s_{1}+s_{2}<-\left|r_{1}-r_{2}\right|$. This proves the proposition when $n=2$. For general $n$ we divide the matrix $B$ in (27) into suitable blocks; then it is easily seen that the result follows from the case $n=2$. Q.E.D.

The theta lifting $\theta\left(\sigma_{\lambda}\right)$ is known in a significant number of cases, in fact it is known in most cases [1, 16, 17]. We can use this together with Proposition 5.1 to exhibit new infinite families of discrete series for the Stiefel manifolds $U(p, q ; D) / U(p-r, q-s ; D)$ for any pair of integers $(r, s)$ with $r \leq p, s \leq$ $q, r+s=n \leq \frac{1}{2}(p+q)$, except when $D=\mathbf{R}, p+q=2 n$ and $p, q$ are both odd (cf. Corollary 3.11). To avoid carrying an extra baggage of notations along, we shall restrict ourselves to the real case for the rest of this section.

So assume $D=\mathbf{R}$. Then $R=0$ and $S$ alone determines $\lambda$. Suppose that $s_{1}>\cdots>s_{k}>0>s_{k+1}>\cdots>s_{n}$ and set $l=n-k$. We assume $k \leq p / 2$ and $l \leq q / 2$. We may assume that $s_{1}, \ldots, s_{n}$ are in fact integers. To the parameter $\lambda$ we may associate a parabolic subalgebra $\mathbf{q}=\mathbf{q}(\lambda)$, of the complexified Lie algebra of $G$. The precise definition of $\mathbf{q}(\lambda)$ is given as in [14]. Let $L(\lambda)$ be the normalizer of $\mathbf{q}$ in $G$. We have

$$
L(\lambda) \simeq T^{n} \times O(p-2 k, q-2 l)
$$

where $T$ is the circle group. Since the Pontrjagin dual of $T^{n}$ is $\mathbf{Z}^{n}$ we may identify the $n$-tuple of integers $\left(s_{1}, \ldots, s_{n}\right)$ with a character of $T^{n}$. This character has a unique extension to $L(\lambda)$, and we will abuse notation to call this $\lambda$. Consider the derived functor module $\mathscr{R}_{\mathbf{q}}^{S}(\lambda)$, as defined in [19] and with normalization also as in [19]. The main result of [14] in the real case is then (see also [1] when $2 n<\min (p, q)$ and [16] when $p, q$ are both even).

Proposition 5.2. Suppose $\left|s_{j}\right| \geq \frac{1}{2}(p+q)-n$ for $j=1, \ldots, n$. Then $\theta\left(\sigma_{\lambda}\right)=$ $\mathscr{R}_{\mathbf{q}} S(\lambda)$

Fix $(r, s)$ with $r \leq p, s \leq q, r+s=n \leq \frac{1}{2}(p+q)$. We want to show that $\mathscr{R}_{\mathbf{q}} S(\lambda)$ is in the discrete series for $O(p, q) / O(p-r, q-s)$ for appropriate $\lambda$. By Proposition 5.1 it suffices to have $k+m_{+}(S) \geq r$ and $l+m_{-}(S) \geq s$. This is easily arranged since $k+l=r+s=n$. We have thus constructed infinite families of discrete series for $O(p, q) / O(p-r, q-s)$.

Consider for example the simplest case $s=0$. We have

Propositon 5.3. Assume that $k \geq l$ and $s_{j}>\left|s_{k+j}\right|$ for $j=1, \ldots, l$. Then the representation $\mathscr{R}_{\mathbf{q}} S(\lambda)$, with $L(\lambda)$ given by $(30)$, occurs in the discrete series of $O(p, q) / O(p-n, q)$.

The family of discrete series for $O(p, q) / O(p-n, q)$ constructed by Kobayashi [12] are all of the form $\mathscr{R}_{\mathbf{q}}^{S}(\lambda)$ where the normalizer of $\mathbf{q}$ in $G$ is of the form $T^{n} \times O(p-2 n, q)$. Thus we have exhibited new infinite families of discrete series even in the case of indefinite Stiefel manifolds $O(p, q) / O(p-n, q)$. Of course similar results are valid in the complex and quaternionic cases. 


\section{THE SKEW-HERMITIAN CASE}

We now state, and prove when necessary, some analogous results in the case when $G$ is the isometry group of a skew-hermitian form. The results run parallel to the hermitian case. We will therefore omit some obvious details.

Suppose the form ( , ) on $V$ is skew-hermitian (and nondegenerate). Let $G=U(V)$ be its isometry group. Let $V_{1} \subseteq V$ be a nondegenerate subspace, and consider the generalized Stiefel manifold $G / G\left(V_{1}^{\perp}\right)$. It is only necessary to consider the case where $D=\mathbf{R}$ or $\mathbf{H}$. Then up to equivalence there is only one nondegenerate skew-hermitian form on $V$. Set $n=\operatorname{dim}_{D} V_{1}$ and let $(,)^{\prime}$ be a nondegenerate hermitian form on $D^{n} \oplus\left(D^{n}\right)^{*}$, so that $D^{n}$ and $\left(D^{n}\right)^{*}$ are both totally isotropic subspaces with respect to $(,)^{\prime}$. Let $G^{\prime}$ be the group of isometries of $(,)^{\prime}$. Analogous to $\S 2$, we have the dual pair $\left(G, G^{\prime}\right)$ and the oscillator representation $\omega$ acting on $L^{2}\left(V^{n}\right)$, where $V^{n}=\operatorname{Hom}\left(D^{n}, V\right)$. Let $P$ be the stabilizer of $D^{n}$ in $G^{\prime}$, with $M \simeq G L(n, D)$ the Levi component. Then $G$ and $P$ act via formulas analogous to (1), (2) and (6). Let $B^{\prime}\left(D^{n}\right)$ denote the space of skew-hermitian forms on $D^{n}$, and define the orbit parameter map $\tau: V^{n} \rightarrow B^{\prime}\left(D^{n}\right)$ as in (5). Set

$$
\Omega=\left\{x \in V^{n} \mid \tau(x) \text { is nondegenerate }\right\} .
$$

Then $\Omega$ is open in $V$, and its complement in $V^{n}$ is a subvariety of positive codimension, hence $L^{2}\left(V^{n}\right)=L^{2}(\Omega)$. Since there is only one equivalence class of nondegenerate skew-hermitian forms on $D^{n}, \Omega$ is a single orbit under $G \times M$. Now one has the obvious analogue of (12), which implies

Theorem 6.1. The restriction of $\omega$ to $G$ is a multiple of $L^{2}\left(G / H_{2}\right)$, where $H_{2}=U\left(V_{1}^{\perp}\right)$.

Corollary 6.2. Let $2 k$ be the smallest even integer satisfying

$$
2 k \geq \frac{1}{2}\left(\operatorname{dim}_{D} V-2+\frac{2}{\operatorname{dim}_{\mathbf{R}} D}\right) .
$$

Let

$$
L^{2}\left(G / H_{2}\right)=\int_{X}^{\oplus} \pi_{x} d \mu(x)
$$

be the direct integral decomposition of $L^{2}\left(G / H_{2}\right)$ into irreducible unitary representations of $G$. Then for almost all $x$, the representation $\pi_{x}$ is strongly $L^{2 k+\varepsilon}$.

Proof. This follows from [13, Theorem 3.2] and Theorem 6.1 in the same say as Theorem 3.7 follows from Lemma 3.3 and Theorem 3.4.

Theorem 6.3. The irreducible subrepresentations $L\left(G / \mathrm{H}_{2}\right)$ are in one-to-one correspondence with the irreducible subrepresentations of $\left.\omega\right|_{G^{\prime}}$ by Howe's duality correspondence. If $\pi$ and $\sigma$ corresponds to each other we write $\pi=\theta(\sigma)$. Then

(a) If $\operatorname{dim}_{D} V \leq 2 n$ then $\pi$ belongs to the discrete series of $G$.

(b) Suppose $\operatorname{dim}_{D} V \geq 2 n$ then the discrete series of $\mathrm{G} / \mathrm{H}_{2}$ is precisely the set

$$
\left\{\theta(\sigma) \mid \sigma \in \widehat{G}_{d}^{\prime}\right\}
$$


Here $\widehat{G}_{d}^{\prime}$ denotes the discrete series representations of $G^{\prime}$.

The proofs are similar to those of $\S \S 2-3$, so we omit them. Note that here the restriction of the oscillator representation to $G^{\prime}$ can also be made into an ordinary representation, hence in the above we spoke of representations of $G^{\prime}$ rather than its two-fold cover.

We remark that by results in [1,16, and 14], most of the representations $\theta(\sigma)$ in Theorem 6.3 are realized as Zuckerman functor modules $\mathscr{R}_{\mathbf{q}} S(\lambda)$, where the normalizer of $\mathbf{q}$ in $G$ is of the form $T^{n} \times S p_{m-2 n}(\mathbf{R})$ in the real case, and $T^{2 n} \times O^{*}(2(m-2 n))$ in the quaternionic case. Here $m$ is the dimension of $V$ over $D$.

\section{REFERENCES}

1. J. Adams, Discrete spectrum of the reductive dual pair $(O(p, q), S p(2 m))$, Invent. Math. 74 (1983), 449-475.

2. $\mathrm{H}$. Anh, Restriction of the principal series of $S L(n, \mathrm{C})$ to some reductive subgroups, Pacific J. Math. 38 (1971), 295-313.

3. D. Barbasch and D. Vogan Jr., The local structure of characters, J. Funct. Anal. 37 (1980), 27-55.

4. _ Primitive ideals and orbital integrals in complex classical groups, Math. Ann. 259 (1982), 153-199.

5. M. Cowling, U. Haagerup, and R. Howe, Almost $L^{2}$ matrix coefficients, J. Reine Angew. Math. 387 (1988), 97-110.

6. J. Diximir, Les C $^{*}$-algebres et leurs representations, Gauther-Villars, Paris, 1964.

7. S. Gelbart, Holomorphic discrete series for the real symplectic group, Invent. Math. 19 (1973), 19-58.

8. R. Howe, Theta series and invariant theory, Proc. Sympos. Pure Math., vol. 33, Amer. Math. Soc., Providence, R.I., 1979.

9. _ Transcending classical invariant theory, J. Amer. Math. Soc. (3) 2 (1989), 535-552.

10. School on Harmonic Analysis, Cortona, 1980.

11. _ Wave front sets of representations of Lie groups, automorphic forms, representation theory, and arithmetic, Papers presented at the Bombay Colloquium, 1979, Tata Institute of Fundamental Research, Bombay, 1981, pp. 117-140.

12. T. Kobayashi, Singular unitary representations and discrete series for indefinite Stiefel manifolds $U(p, q ; \mathbf{F}) / U(p-m, q ; \mathbf{F})$, preprint.

13. J. S. Li, Singular unitary representations of classical groups, Invent. Math. 97 (1989), 237-255.

14. _ Theta lifting for unitary representations with non-zero cohomology, Duke Math. J. (3) 61 (1990).

15. T. Matsuki and T. Oshima, A description of discrete series for semi-simple symmetric spaces, Adv. Stud. Pure Math., no. 4, Academic Press, 1984, pp. 331-390.

16. C. Moeglin, Correspondance de Howe pour les paires reductive duales, quelques calculs dans le cas Archimedien, preprint.

17. S. Rallis and G. Schiffmann, Weil representation. I. Intertwining distributions and discrete spectrum, Mem. Amer. Math. Soc., vol. 25, no. 231, 1980.

18. $\mathrm{H}$. Schlichtkrull, $A$ series of unitary irreducible representations induced from a symmetric subgroup of a semi-simple Lie group, Invent. Math. 68 (1982), 497-516. 
19. D. Vogan, Unitary representations of real reductive groups, Ann. of Math. Studies, vol. 118, Princeton Univ. Press, Princeton, N.J, 1987.

20. __ Irreducibility of discrete series representations for semi-simple symmetric spaces, Adv. Stud. Pure Math., no. 14, Academic Press, 1988, pp. 191-221.

Department of Mathematics, University of Maryland, College Park, Maryland 20742

E-mail address: jsl@karen.umd.edu 